\title{
Long-lasting effects of yohimbine on the ejaculatory function in male dogs
}

\author{
Akihiko Yonezawa ${ }^{1}$, Masaru Yoshizumi ${ }^{1}$, Mamoru Ebiko ${ }^{1}$, Toshiyasu Amano ${ }^{2}$, Yukio Kimura ${ }^{3}$ and Shinobu \\ SAKURADA ${ }^{1}$ \\ ${ }^{1}$ Department of Physiology and Anatomy, Tohoku Pharmaceutical University, 4-4-1 Komatsushima, Aoba-ku, Sendai 981-8558; \\ ${ }^{2}$ Department of Urology, Nagano Red Cross Hospital, 5-22-1 Wakasato, Nagano 380-8582; and ${ }^{3}$ Towada Urology Hospital, 1-3-8 \\ Motomachi-Higashi, Towada 034-0003, Japan
}

(Received 2 August 2005; and accepted 19 August 2005)

\begin{abstract}
Previous studies have demonstrated that systemic administration of a low dose of the alpha2-adrenoceptor antagonists stimulates the ejaculatory response of male dogs, when this function is analyzed using the amount of ejaculated semen in response to genital stimulation. The present study was designed to further examine the features of the stimulatory effects of the alpha2-adrenoceptor antagonists on ejaculation, especially the duration of action. Treatment with yohimbine $(0.1 \mathrm{mg} /$ $\mathrm{kg}$, i.p.) to male dogs, at $0.5,1,3$, or $5 \mathrm{~h}$ before the testing, produced a significant stimulatory effects on the ejaculatory response elicited by manual penile stimulation; the amount of ejaculated semen was increased and the onset of ejaculation was shortened following each treatment. However, such effects were not observed in the treatment with yohimbine at 8 and $24 \mathrm{~h}$ before the testing, indicating that the ejaculatory stimulation induced by yohimbine lasted for a relative long period. By contrast, the stimulatory effects of RX821002 (0.1 mg/kg, i.p.), a selective alpha2-adrenoceptor antagonist, on ejaculation were observed only for $1 \mathrm{~h}$ after administration. To determine the contribution of the alpha2-adrenoceptor blockade for the long-lasting effect of yohimbine, we tested whether yohimbine can prevent the ejaculatory inhibition induced by clonidine, an alpha2adrenoceptor agonist. The ejaculatory inhibition (a decrease in the amount of ejaculated semen and a delay onset of ejaculation) elicited by clonidine $(0.05 \mathrm{mg} / \mathrm{kg}$, i.p.; $1 \mathrm{~h}$ before testing) was completely blocked by pretreatment with yohimbine at 1 or $5 \mathrm{~h}$ before the testing, whereas the pretreatment with the drug at $24 \mathrm{~h}$ before the testing did not affect the clonidine-induced ejaculatory inhibition. These results indicate that yohimbine-induced ejaculatory stimulation is continued for a relative long period (at least $5 \mathrm{~h}$ after administration), and this long-lasting effects may be related to the alpha2-adrenoceptor blocking property of the drug.
\end{abstract}

Research has indicated that central alpha2-adrenergic mechanisms may be involved in regulation of male sexual performance. A number of studies have demonstrated that the compounds with central

Address correspondence to: Akihiko Yonezawa, Ph.D., Department of Physiology and Anatomy, Tohoku Pharmaceutical University, 4-4-1 Komatsushima, Aoba-ku, Sendai 981-8558, Japan

Tel: +81-22-234-4181, Fax: +81-22-275-2013

E-mail: yonezawa@tohoku-pharm.ac.jp alpha2-adrenoceptor blocking action can facilitate both sexual arousal and copulatory events associated with male sexual behavior $(5-7,10-11)$. With regard to male sexual function especially ejaculation, however, pharmacological research has been hampered by the lack of a suitable animal model. We have previously established a dog model, which analyzes using the amount of ejaculated semen in response to the genital stimulation as an index for a quantitative assessment of the ejaculatory capacity (15). Using this model, we reported for the first 
time that systemically administered yohimbine, an alpha2-adrenoceptor antagonist, affects the ejaculatory capacity of dogs in a biphasic fashion; low doses $(0.01-0.3 \mathrm{mg} / \mathrm{kg}$, i.p.) increase the amount of ejaculated semen, whereas higher doses $(>1.0 \mathrm{mg} /$ $\mathrm{kg}$, i.p.) diminish the ejaculatory capacity (15). Moreover, we clearly demonstrated that yohimbine, at a low dose $(0.1 \mathrm{mg} / \mathrm{kg}$, i.p.), can prevent and reverse the diminished ejaculatory capacity during a period of frequent ejaculation $(16,19)$. A similar results was also obtained by other alpha2-adrenoceptor antagonists that act in both the central and the peripheral nervous system, but not the peripheral nervous system only (17), indicating that central alpha2-adrenoceptors may be involved in the modulation of the ejaculatory process. The clinical trials enforced on the basis of these results have shown that treatment with yohimbine is effective for the patients with ejaculatory dysfunction, which accompanies by the diminished ejaculatory capacity $(1,4)$.

The present study was undertaken to determine the features of the stimulatory effect of alpha2-adrenoceptor antagonists, such as yohimbine and RX821002, on ejaculation in male dogs, especially the duration of action. Furthermore, we also examined the putative pharmacological interaction of yohimbine with alpha2-adrenergic mechanism in the stimulation of the ability of ejaculation.

\section{MATERIALS AND METHODS}

Animals. Nine adult male beagle dogs weighing 13 to $20 \mathrm{~kg}$ were used for the study in various pharmacological experiments. Prior to the experiments, all animals were tested for the reliable occurrence of ejaculation and penile erection in response to the manual penile stimulation. Dogs were individually housed in a room that was lit between 6:00 a.m. and $8: 00 \mathrm{p} . \mathrm{m}$. and maintained at a temperature $\left(22-24^{\circ} \mathrm{C}\right)$ and relative humidity $(50-60 \%)$. Water and standard dog food (CD-1, CLEA, Japan) were available at all times except during the experimental sessions. This study was reviewed and approved by the Animal Committee of Tohoku Pharmaceutical University.

Drugs. Drugs used in this study were yohimbine $\mathrm{HCl}$ (Nakarai Tesque, Kyoto, Japan), RX821002 $\mathrm{HCl}$ (Research Biochemicals International, Wayland, MA, USA) and clonidine $\mathrm{HCl}$ (Sigma, St. Louis, MO, USA). Yohimbine was dissolved in sterile distilled water; RX821002 and clonidine in sterile saline $(0.9 \% \mathrm{NaCl})$. All drugs were prepared immediately prior to testing and were injected intraperitoneally (i.p.) in a volume of $0.2 \mathrm{~mL} / \mathrm{kg}$ body weight. The doses of the drugs were calculated as the salt.

Testing procedure. The testing procedure for evaluating the drug effects on male sexual functions were similar to that employed previously (15). For all experimental sessions, animals were transferred to an experimental room 15-30 min before the testing and then injected the appropriate drugs or vehicle. Ejaculation and penile erection were elicited by continuous manual stimulation (for $5 \mathrm{~min}$ ) of the penis, which applied light pressure and gently rubbed the body of the penis just behind the bulbus glandis. This stimulation could easily produce a rapid onset of ejaculation $(<15 \mathrm{sec})$, and the intermittent ejection of semen occurred subsequently when the stimulation was continued. A rapid penile tumescence and pelvic thrusting behavior also simultaneously occurred by the stimulation, and all animals showed full erection within $1 \mathrm{~min}$ after the start of the stimulation. To assess ejaculation, the following parameters were recorded: (1) the amount of ejaculated semen collected during a period of the stimulation, (2) the time from a start of the stimulation to the first ejaculation (ejaculation latency). Manual penile stimulation and observation of ejaculation parameters were carried out by the same observer.

Experiment 1 : Each animal served as his own control, because the amount of ejaculated semen produced by manual penile stimulation varied considerably among individual animals (15). To determine the basal ejaculate value (pre-drug value), the semen collection was made 5 times every 4 days prior to the testing and the mean weight of ejaculates was calculated in each animal. After the determination of the pre-drug value, each dog was administered vehicle or each alpha2-adrenoceptor antagonist. All data for ejaculated semen were represented as a percentage of the pre-drug value. The experimental sessions on a given animals were always 4 day intervals.

Experiment 2 : Experiment for the effects of yohimbine on clonidine-induced ejaculatory inhibition was carried out for at least 4 weeks after Experiment 1. Each animal was retested and recalculated the basal ejaculate value (pre-drug value). The experimental sessions on a given animals were 7-10 day intervals.

Data analysis. Nonparametric statistics were used throughout. Overall differences were analyzed using 
the Friedman two-way analysis of variance. When significant differences $(p<0.05)$ were obtained, the Wilcoxon-matched pairs signed-ranks test was applied to identify significant differences between the treatments. Data are presented as the mean \pm S.E.M.

\section{RESULTS}

\section{Ejaculation induced by manual penile stimulation}

In control experiment, the reliable ejection of semen occurred within $10 \mathrm{sec}$ after the start of manual penile stimulation, and subsequently occurred intermittently when the stimulation was continued. The weight of ejaculated semen produced by the stimulation (for $5 \mathrm{~min}$ ) varied considerably among individual dogs, as previously reported $(15,17)$. The basal value (pre-drug value) in each animal obtained by regular semen collection ( 5 times at 4 day intervals) ranged from 4.35 to $10.27 \mathrm{~g}$. However, the time to time variations in the weight of ejaculated semen is little changed in each of 9 dogs. The coefficients of variation within animals ranged from 5.6 to $12.1 \%$ with an average of $9.2 \%$. The results of the vehicle-treated animals show that the ejaculatory responses were stably produced by the manual penile stimulation (Fig. 1).

\section{Experiment 1: Time-course effects of yohimbine on ejaculation in dogs}

Fig. 1 shows the time-course effects of yohimbine on the ejaculatory response elicited by manual penile stimulation in dogs. Treatment with yohimbine $(0.1 \mathrm{mg} / \mathrm{kg}$, i.p. $)$, at $0.5,1,3$, or $5 \mathrm{~h}$ before the testing, produced a stimulatory effect on the ejaculatory response in a same degree; the amount of ejaculated semen produced by the stimulation was significantly increased and the onset of the first ejaculation was significantly shortened following each treatment. However, such effect was not observed in the treatment with yohimbine at 8 or $24 \mathrm{~h}$ before the testing. In contrast, the stimulatory effect of RX821002 $(0.1 \mathrm{mg} / \mathrm{kg}$, i.p.), a selective alpha2-adrenoceptor antagonist, on ejaculation was observed only at 0.5 and $1 \mathrm{~h}$ after administration (Fig. 2). Full erection induced by the stimulation was obtained following above all treatments.
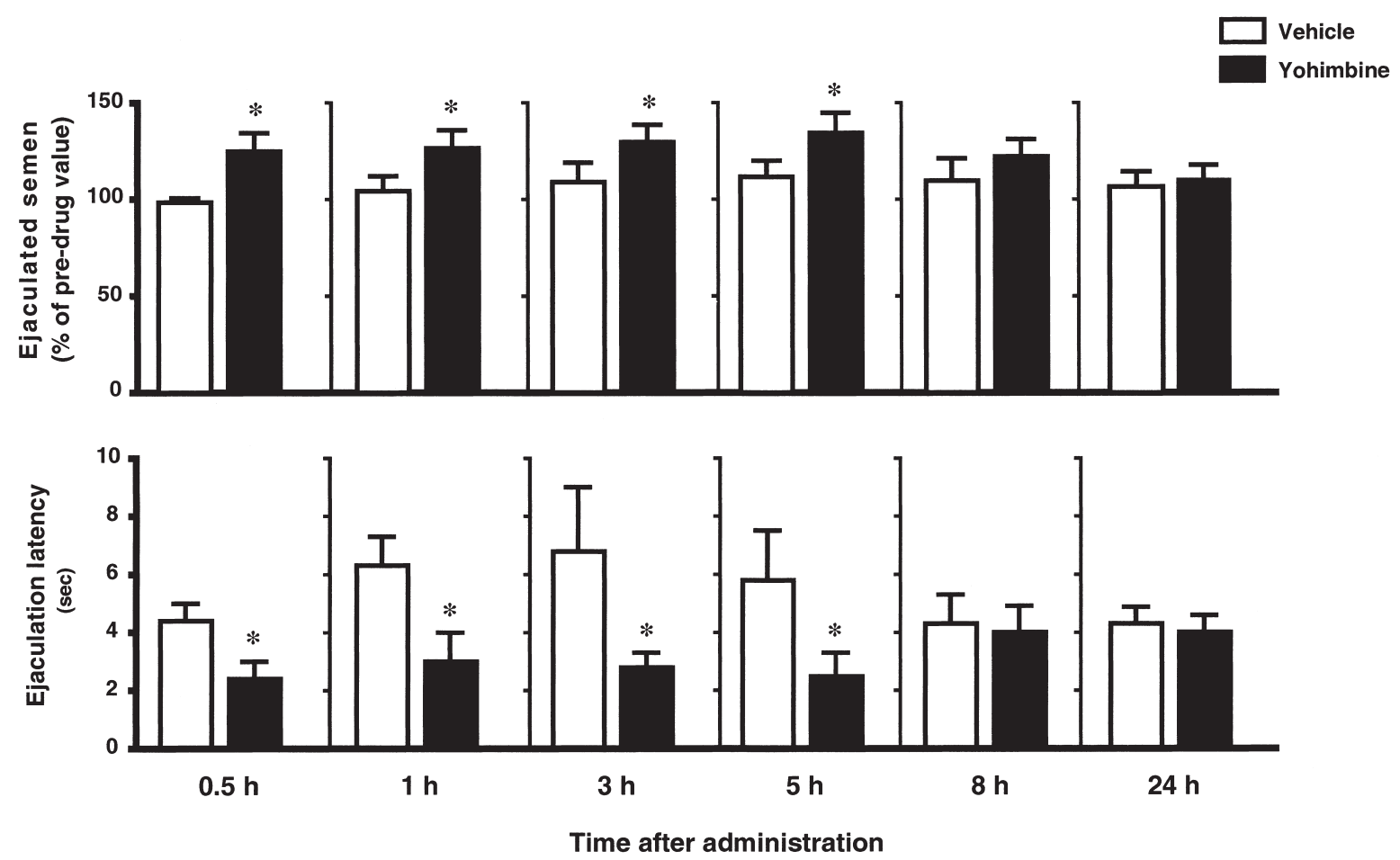

Fig. 1 Effect of systemic administration of yohimbine at various times on the ejaculatory response elicited by manual penile stimulation in dogs. Yohimbine $(0.1 \mathrm{mg} / \mathrm{kg})$ was injected i.p. $0.5,1,3,5,8$ and $24 \mathrm{~h}$ before the testing, respectively. Ejaculated semen was collected with a pre-weighed plastic beaker during the period of the stimulation (for 5 min) and then weighed on an electro-milibalance. Ejaculation latency was measured by stop-watch. Each values are mean \pm S.E.M. of 9 animals in separate experiments. Time interval between treatment with vehicle and yohimbine in the same animals was 4 days. ${ }^{*} P<0.05$ when compared to vehicle-treated animals. 

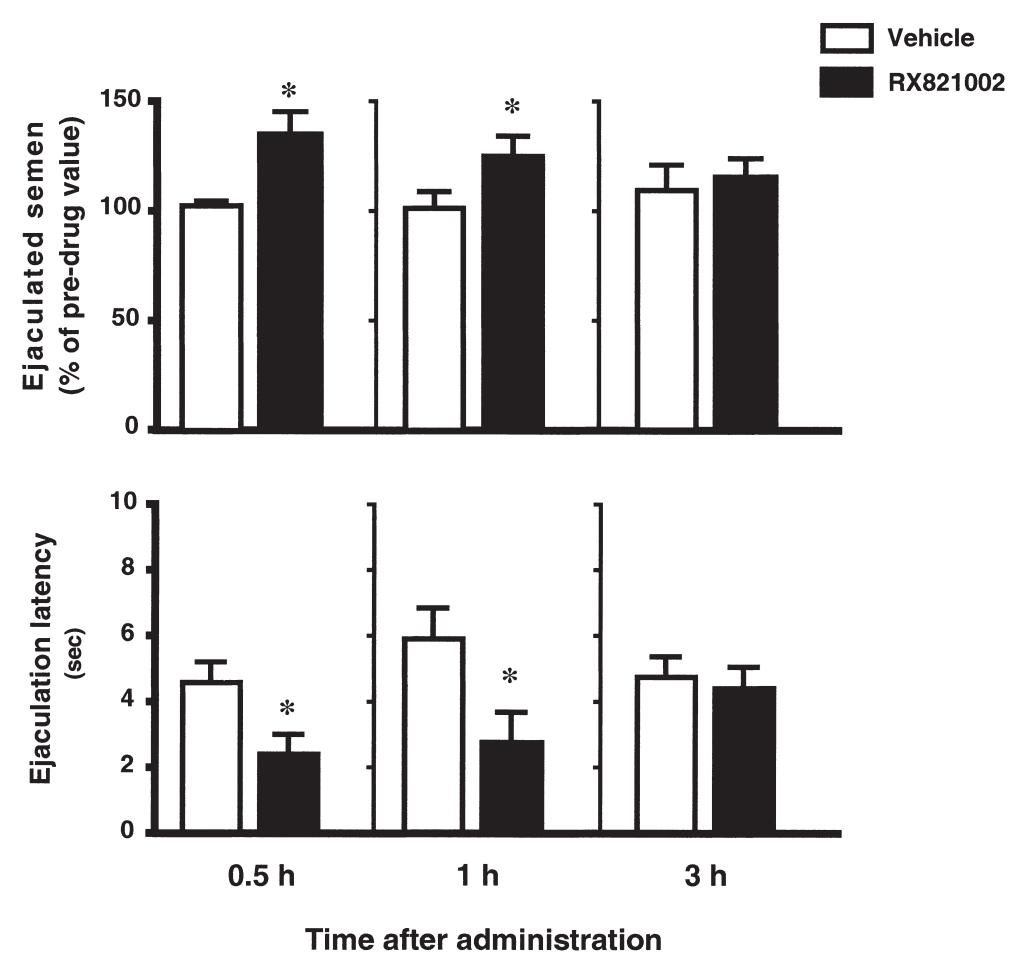

Fig. 2 Effect of systemic administration of RX821002 at various times on the ejaculatory response elicited by manual penile stimulation in dogs. RX821002 $(0.1 \mathrm{mg} / \mathrm{kg})$ was injected i.p. $0.5,1$, and $3 \mathrm{~h}$ before the testing, respectively. Ejaculated semen was collected with a pre-weighed plastic beaker during the period of the stimulation (for 5 min) and then weighed on an electro-milibalance. Ejaculation latency was measured by stop-watch. Each values are mean \pm S.E.M. of 9 animals in separate experiments. Time interval between treatment with vehicle and RX821002 in the same animals was 4 days. ${ }^{*} \mathrm{P}<0.05$ when compared to vehicle-treated animals.

Experiment 2: Involvement of alpha2-adrenoceptor blockade on the ejaculatory stimulation induced by yohimbine

To determine the contribution of the alpha2-adrenoceptor blockade to the long-lasting effect of yohimbine, we tested whether yohimbine can prevent the ejaculatory inhibition induced by clonidine, an alpha2-adrenoceptor agonist (Fig. 3). Systemic administration of clonidine $(0.05 \mathrm{mg} / \mathrm{kg}$, i.p.; $1 \mathrm{~h}$ before the testing) produced an ejaculatory inhibition such as a decrease in the amount of ejaculated semen and a delay onset in ejaculation latency. This inhibition induced by clonidine was completely blocked by pretreatment with yohimbine at 1 and $5 \mathrm{~h}$ before the testing, whereas the pretreatment with the drug at $24 \mathrm{~h}$ before the testing did not block the clonidine-induced ejaculatory inhibition.

\section{DISCUSSION}

In the present study, we replicated previous work in our laboratory indicating that systemic administration of a low dose of yohimbine and RX821002 stimulates the ejaculatory response induced by manual penile stimulation in dogs $(15,17)$. In fact, both of an increase in the amount of ejaculated semen and a shortening in the ejaculatory latency were observed by these compounds. There is both direct and indirect evidence that the stimulatory effect of yohimbine and RX821002 on ejaculation is mediated through the blockade of the alpha2-adrenoceptors in the central nervous system. Thus, the ejaculatory inhibition in dogs induced by centrally or systemically administered clonidine, an alpha2-adrenoceptor agonist, was completely antagonized by pretreatment with systemic administration of yohimbine (18). Furthermore, the selective alpha2-adrenoceptor antagonists such as rauwolscine, idazoxan and RX821002, which possess the blocking activity on both central and peripheral alpha2-adrenoceptors, have also a stimulatory effect on ejaculation (i.e., increase in the amount of ejaculated semen), whereas a peripherally acting alpha2-adrenoceptor antagonist did not affect the ejaculatory capacity in dogs (17).

An interesting finding in the present study is that 


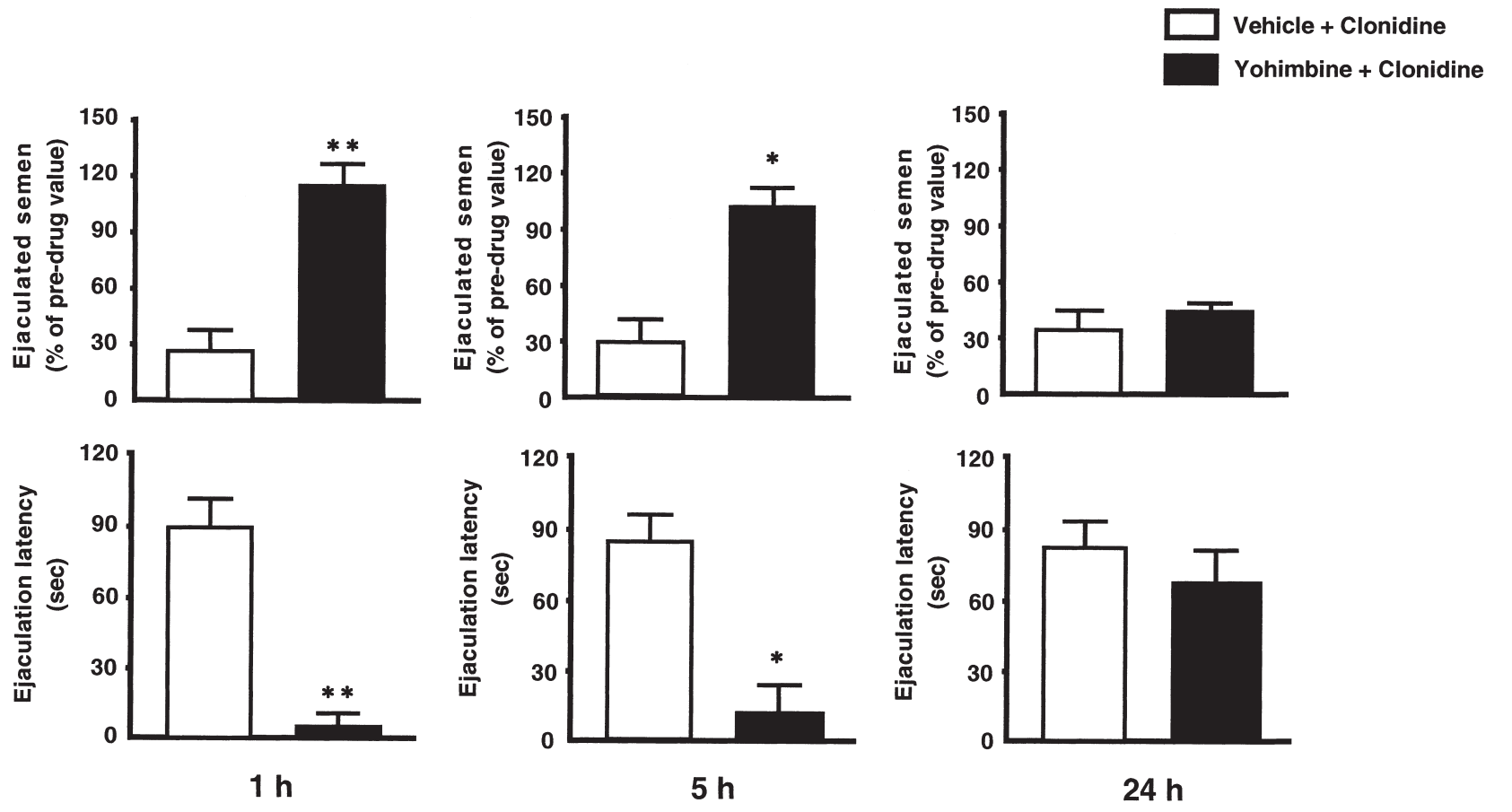

Fig. 3 Effect of pretreatment with yohimbine on the ejaculatory inhibition induced by clonidine. Yohimbine $(0.1 \mathrm{mg} / \mathrm{kg}) \mathrm{was}$ injected i.p. 1, 5 or $24 \mathrm{~h}$ before the testing and clonidine $(0.05 \mathrm{mg} / \mathrm{kg})$ was injected i.p. $1 \mathrm{~h}$ before the testing. Ejaculated semen was collected with a pre-weighed plastic beaker during the period of the stimulation (for 5 min) and then weighed on an electro-milibalance. Ejaculation latency was measured by stop-watch. Each values are mean \pm S.E.M. of 9 animals in separate experiments. Time interval between treatment with vehicle and yohimbine in the same animals was 7-10 days. ${ }^{\star *} \mathrm{P}<0.01,{ }^{*} \mathrm{P}<0.05$ when compared to vehicle-treated animals.

the stimulatory effect of yohimbine on ejaculation was longer lasting than that of RX821002. In fact, both of an increase in the amount of ejaculated semen and a shortening in the ejaculatory latency induced by yohimbine can be detected until at least $5 \mathrm{~h}$ after administration, whereas RX821002-induced ejaculatory stimulation is only continued for $1 \mathrm{~h}$ after administration. These results indicate that systemic administration of yohimbine can affect the ejaculatory function for a relative long period (at least $5 \mathrm{~h}$ after administration). The difference on the duration of action between yohimbine and RX821002 was also reported in other canine functions, including food intake (2).

Although there is no data concerning the pharmacokinetic for yohimbine in dogs, the analysis in human subjects has clearly indicated that yohimbine is rapidly absorbed and eliminated from the plasma (9) (absorption half-time $0.17 \pm 0.11 \mathrm{~h}$ and elimination half-life $0.6 \pm 0.26 \mathrm{~h}$ ). The data of urinary excretion and partitioning into red blood cells revealed that the rapid plasma clearance of yohimbine may result from the metabolism (a first-pass effect) but not from the renal elimination or sequestration by red blood cells (9). Based on these findings, it is probable that the stimulatory effect of yohimbine on ejaculation in dogs is related to an active metabolite(s) of the compound.

It has been shown that hepatic metabolism produces two hydroxylated metabolites of yohimbine, 10-OH-yohimbine and 11-OH-yohimbine (8). Le Verge et al. (8) have demonstrated that 11-OH-yohimbine metabolite is largely present in the plasma and exhibits a longer elimination half-life than parent compound (11-OH-yohimbine $6 \mathrm{~h}$ vs yohimbine $1 \mathrm{~h})$. In subsequent pharmacological study, it has been shown that 11-OH-yohimbine possesses the alpha2-adrenoceptor antagonist properties (3). In the present study, the ejaculatory inhibition induced by clonidine, the alpha2-adrenoceptor agonist, was almost equivalently blocked by pretreatment with yohimbine at 1 or $5 \mathrm{~h}$ after administration, respectively. These results suggest the possibility that the longlasting effects of yohimbine on ejaculation may be related to the alpha2-adrenoceptor blocking property of yohimbine and/or its hepatic metabolites, includ- 
ing 11-OH-yohimbine. Further investigation is, however, necessary to elucidate this consideration.

In conclusion, the present study indicates that yohimbine-induced ejaculatory stimulation is continued for a relative long period (until at least $5 \mathrm{~h}$ after administration), and that the long-lasting effects of yohimbine may be related to the alpha2-adrenoceptor blocking property of yohimbine and/or its active metabolites.

\section{REFERENCES}

1. Amano T, Kobori Y, Matsui F, Takemae K and Yonezawa A (2002) Treatment for ejaculation dysfunction with the administration of yohimbine. Jap J Impot Res 17, 225-228.

2. Berlan M, Galitzky J, Tran MA and Montastruc P (1991) Anorectic effect of alpha 2-antagonists in dog: effect of acute and chronic treatment. Pharmacol Biochem Behav 39, 313320.

3. Berlan M, Le Verge R, Galitzky J and Le Corre P (1993) Alpha 2-adrenoceptor antagonist potencies of two hydroxylated metabolites of yohimbine. Br J Pharmacol 108, 927-932.

4. Brindley GS (1994) Impotence and ejaculatory failure. In: Handbook of Neuro-Urology (Rushton DN, ed.), pp329-348, Marcel Dekker, Inc., New York.

5. Clark JT, Smith ER and Davidson JM (1984) Enhancement of sexual motivation in male rats by yohimbine. Science 225, 847-849.

6. Clark JT, Smith ER and Davidson JM (1985) Testosterone is not required for the enhancement of sexual motivation by yohimbine. Physiol Behav 35, 517-521.

7. Clark JT, Smith ER and Davidson JM (1985) Evidence for the modulation of sexual behavior by $\alpha$-adrenoceptors in male rats. Neuroendocrinology 41, 36-43.

8. Le Verge R, Le Corre P, Chevanne F, Doe De Maindreville M, Royer D and Levy J (1992) Determination of yohimbine and its two hydroxylated metabolites in humans by high-performance liquid chromatography and mass spectral analysis. $J$ Chromatogr 574, 283-292.
9. Owen JA, Nakatsu SL, Fenemore J, Condra M, Surridge DH and Morales A (1987) The pharmacokinetics of yohimbine in man. Eur J Clin Pharmacol 32, 577-582.

10. Sala M, Braida D, Leone MP, Calcaterra P, Monti S and Gori E (1990) Central effect of yohimbine on sexual behavior in the rat. Physiol Behav 47, 165-173.

11. Smith ER and Davidson JM (1990) Yohimbine attenuates aging-induced sexual deficiencies in male rats. Physiol Behav 47, 631-634.

12. Smith ER, Lee RL, Schnur SL and Davidson JM (1987) $\alpha 2$ Adrenoceptor antagonists and male sexual behavior: I. Mating behavior. Physiol Behav 41, 7-14.

13. Smith ER, Lee RL, Schnur SL and Davidson JM (1987) $\alpha 2-$ Adrenoceptor antagonists and male sexual behavior: II. Erectile and ejaculatory reflexes. Physiol Behav 41, 15-19.

14. Tallentire D, McRae G, Spedding M, Clark R and Vickery B (1996) Modulation of sexual behavior in the rat by a potent and selective $\alpha 2$-adrenoceptor antagonist, delequamine (RS-15385-197). Br J Pharmacol 118, 63-72.

15. Yonezawa A, Kawamura S, Ando R, Tadano T, Nobunaga $T$ and Kimura Y (1991) Biphasic effects of yohimbine on the ejaculatory response in the dog. Life Sci 48, PL 103-109.

16. Yonezawa A, Kawamura S, Ando R, Tadano T, Nobunaga $T$ and Kimura Y (1991) Diminution of ejaculatory capacity induced by frequent ejaculation in dogs: Prevention and reversal by yohimbine. Andrologia 23, 71-74.

17. Yonezawa A, Ando R, Watanabe C, Furuta S, Kutsuwa M, Sakurada S and Kimura Y (2001) $\alpha 2$-Adrenoceptor antagonists: effects on ejaculation, penile erection and pelvic thrusting behavior in dogs. Pharmacol Biochem Behav 70, 141147.

18. Yonezawa A, Ando R, Tadano T, Kisara K, Miyamoto A and Kimura Y (1986) Evidence for central $\alpha 2$-adrenergic mechanism of clonidine-induced ejaculatory disturbance in dogs. $J$ Pharmacobiodyn 9, 1032-1035.

19. Yonezawa A, Kutsuwa M, Amano T, Kimura Y and Sakurada S (2003) Comparison of the effects of yohimbine, naloxone and 8-OH-DPAT on the diminished ejaculatory capacity induced by repeated ejaculation. Biomed Res 24, 71-75. 